\title{
QUANTUM GEOMETRY OF NONCOMMUTATIVE BERNOULLI SHIFTS
}

\author{
ROBERT ALICKI \\ Institute of Theoretical Physics and Astrophysics, University of Gdańsk \\ Wita Stwosza 57, 80-952 Gdańsk, Poland \\ E-mail: fizra@univ.gda.pl
}

\begin{abstract}
We construct an example of a noncommutative dynamical system defined over a two dimensional noncommutative differential manifold with two positive Lyapunov exponents equal to $\ln d$ each. This dynamical system is isomorphic to the quantum Bernoulli shift on the half-chain with the quantum dynamical entropy equal to $2 \ln d$. This result can be interpreted as a noncommutative analog of the isomorphism between the classical one-sided Bernoulli shift and the expanding map of the circle and moreover as an example of the noncommutative Pesin theorem.
\end{abstract}

1. Introduction. This paper is motivated by the standard example of a metric isomorphism between two classical dynamical systems: the one sided $\left(\frac{1}{d}, \ldots, \frac{1}{d}\right)$-Bernoulli scheme and the expanding map of the circle $z \mapsto z^{d}[7]$.

The (one-sided) Bernoulli shift is an example of a system studied in the ergodic theory based on probability spaces with measure preserving endomorphisms. The chaotic properties are quantitatively described by a single parameter - the Kolmogorov-Sinai entropy which is equal to $\ln d$. On the other hand the circle $\{z \in \mathbf{C} ;|z|=1\}$ possesses a differential structure compatible with the Lebesgue measure. The expanding map is smooth and its chaotic properties are indicated by the positive Lyapunov exponent which is equal to $\ln d$ (and as expected equal to K-S entropy) in this case.

The natural quantum generalization of the $\left(\frac{1}{d}, . ., \frac{1}{d}\right)$-Bernoulli scheme is given by a shift acting on the infinite spin half-chain with a tracial state. This is an example of a noncommutative $C^{*}$-algebraic dynamical system with a quasi-local $C^{*}$-algebra of observables obtained as an infinite tensor product of the single-site $d \times d$-matrix algebras and a shift invariant state. The models of this type with general shift-invariant states have been discussed in numerous papers and their different ergodic properties were studied in de-

1991 Mathematics Subject Classification: Primary 46L55; Secondary 46L87.

Research of the author supported by KBN grant 2 PO3B 144-09.

The paper is in final form and no version of it will be published elsewhere. 
tail. Among others, the noncommutative generalization of the Kolmogorov-Sinai entropy proposed and developed in the references $[2,3,4,12]$ can be applied and yields the value $2 \ln d$. The present paper deals with the following question: is it possible to construct a noncommutative dynamical system which is isomorphic in a certain sense to the model discussed above and moreover possesses a noncommutative differential structure which supports quantum Lyapunov exponents? We expect also that the sum of positive Lyapunov exponents should be equal to $2 \ln d$. In Section 4 we shall give a positive answer to this question constructing the noncommutative expanding map acting on a two dimensional "quantum manifold" and with two positive Lyapunov exponents each of them equal to $\ln d$. For a completely different example of quantum expanding map see ref. [1].

2. Quantum Bernoulli scheme and its dynamical entropy. A discrete-time noncommutative $C^{*}$-algebraic dynamical system is described in terms of a unital $C^{*}$ algebra $\mathcal{A}$, an endomorphism $\Theta$ of $\mathcal{A}$, and a state $\omega$, invariant under $\Theta$. In the following we shall use a particular quantum analog of the Kolmogorov-Sinai entropy presented in our previous papers $[2,3,4,12]$ (see also [10]) and named Alf entropy in honor of the nice alien hero of the TV series. As an additional structure we need a specified unital *-subalgebra $\mathcal{A}_{0} \subset \mathcal{A}$, globally invariant under $\Theta$, which represents "local" or "smooth" observables of the system. The fundamental object is the operational partition of unity $\mathcal{X}=\left(x_{i} ; 1 \leq i \leq k\right)$ which consists of elements of $\mathcal{A}$ satisfying:

$$
\sum_{i=1}^{k} x_{i}^{*} x_{i}=\mathbf{1} \text {. }
$$

Two partitions $\mathcal{X}=\left\{x_{i} ; 1 \leq i \leq k\right\}$ and $\mathcal{Y}=\left\{y_{j} ; 1 \leq j \leq l\right\}$ produce a new partition $\mathcal{X} \circ \mathcal{Y}=\left\{x_{i} y_{j} ; 1 \leq i \leq k, 1 \leq j \leq l\right\}$ and the time evolution of the partition $\mathcal{X}$ gives the partition $\Theta(\mathcal{X})=\left\{\Theta\left(x_{i}\right) ; 1 \leq i \leq k\right\}$.

A given partition $\mathcal{X}$ of size $k$ defines a $k \times k$ density matrix $\rho[\mathcal{X}]$ with $(i, j)$ matrix element equal to $\omega\left(x_{j}^{*} x_{i}\right)$. The dynamical entropy is constructed in terms of the usual von Neumann entropy for the density matrix $S(\rho)=-\operatorname{Tr} \rho \ln \rho$ as

$$
h_{\left(\mathcal{A}_{0}, \Theta, \omega\right)}=\sup _{\mathcal{X} \subset \mathcal{A}_{0}}\left\{\limsup _{m} \frac{1}{m} S\left(\rho\left[\Theta^{m-1}(\mathcal{X}) \circ \cdots \circ \Theta(\mathcal{X}) \circ \mathcal{X}\right]\right)\right\} .
$$

We consider a quantum spin half-chain with a single site algebra $\mathcal{M}_{d}$ of $d \times d$ matrices. The dynamics $\Lambda$ is given by the right shift and the state is now a canonical normalized trace $\tau$ on the quasi-local algebra $\mathcal{U}=\left(\mathcal{M}_{d}\right)^{\mathrm{N}}$. Choosing for the subalgebra $\mathcal{A}_{0}$ the strictly local algebra $\mathcal{U}_{0}$, which consists of observables involving only a finite number of spins, one obtains [3]

$$
h_{\left(\mathcal{U}_{0}, \Lambda, \tau\right)}=2 \ln d .
$$

It is interesting that the other approaches to noncommutative dynamical entropy yield quite different results. Namely, the CNT entropy can be computed for this model too and is equal to $\ln d[6]$, the same value has been obtained for the Voiculescu growth-entropy [13] and the Hudetz "hybrid" entropy [9]. The Alf entropy depends on the additional structure of local or smooth observables. However, its numerical value does not change if we replace the local algebra by the algebra of smooth elements defined, for example, 
in terms of oscillation norms [12]. On the other hand working within the $W^{*}$-algebraic scheme and taking as $\mathcal{A}_{0}$ the full von Neumann algebra of the dynamical system one obtains typically an infinite Alf entropy. This suggests deeper connections between our dynamical entropy and the noncommutative geometry of quantum dynamical systems.

3. Quantum Lyapunov exponents. There were several attempts to define quantum Lyapunov exponents $[8,11]$. In the abstract context of a $C^{*}$-algebraic dynamical system $(\mathcal{A}, \Theta)$ with the additional structure of unbounded derivations $\left\{\mathcal{D}_{i}\right\}$ over a $\Theta$-invariant smooth subalgebra $\mathcal{A}_{\infty} \subset \mathcal{A}$ the Lyapunov exponents can be defined as [8]

$$
\lambda_{i}(X)=\lim _{n \rightarrow \infty} \frac{1}{n} \ln \left\|\mathcal{D}_{i}\left(\Theta^{n}(X)\right)\right\|, \quad X \in \mathcal{A}_{\infty} .
$$

In the interesting cases $\lambda_{i}(X)$ should take only a finite number of values. However, in a general situation there exist no canonical derivations which could span a noncommutative analog of the tangent space. The nice special case is the noncommutative torus given by the irrational rotation algebra $\mathcal{A}_{\theta}$ generated by two unitaries and possessing a corresponding two dimensional "tangent space" of outer derivations [8]. In the example discussed below such a natural system of derivations has not been found and instead we introduce a weaker geometrical structure supporting the notion of Lyapunov exponents. Namely, we assume the existence of a nonnegative bilinear form on $\mathbf{C}^{\nu} \otimes \mathcal{A}_{\infty}$ denoted by $\Delta(F, G) ; F, G \in \mathbf{C}^{\nu} \otimes \mathcal{A}_{\infty}$. Here $\mathbf{C}^{\nu}$ may be treated as a (complexified) model of the "tangent space". For any $\xi \in \mathbf{C}^{\nu}$ and $X \in \mathcal{A}_{\infty}$ we can define the associated Lyapunov exponent

$$
\lambda(\xi, X)=\lim _{n \rightarrow \infty} \frac{1}{2 n} \ln \left\{\Delta\left(\xi \otimes \Theta^{n}(X), \xi \otimes \Theta^{n}(X)\right)\right\} .
$$

One can expect that similarly to the classical case $\mathbf{C}^{\nu}$ can be decomposed into one dimensional subspaces corresponding to a finite sequence $\left\{\lambda_{i}\right\}$ of Lyapunov exponents which are independent on $X$. In the cases for which there exists a canonical set of derivations $\left\{\mathcal{D}_{j}\right\}$ we put

$$
\Delta\left(e_{j} \otimes X, e_{k} \otimes Y\right)=\omega\left(\mathcal{D}_{j}\left(X^{*}\right) \mathcal{D}_{k}(Y)\right)
$$

where $\left\{e_{j}\right\}$ is a linear basis in $\mathbf{C}^{\nu}$ and $\omega$ is a $\Theta$-invariant state on $\mathcal{A}$.

4. Quantum expanding maps. We begin with some preliminary definitions and notation. Fix an orthonormal basis $\left\{e_{1}, \ldots, e_{d}\right\}$ of $\mathbf{C}^{d}$ and consider two unitary matrices $u$ and $v$ defined by the action on the basis

$$
u e_{j}=\gamma^{j} e_{j} \text { and } v e_{j}=e_{j+1}
$$

where $\gamma$ is the $d^{\text {th }} \operatorname{root} \exp (2 \pi i / d)$ of 1 and where the subscript $d+1$ is identified with 1. It is easy to check that $u$ and $v$ satisfy the commutation relation

$$
u v=\gamma v u
$$

and $u^{d}=v^{d}=1$. An elementary proof shows that $\{u, v\}$ generate the full matrix algebra $M_{d}$. In the following we shall use the exponential form

$$
u=e^{i a}, v=e^{i b} .
$$


We return to the one-sided quantum Bernoulli system $(\mathcal{U}, \Lambda, \tau)$ described in Section 2 . We define now two elements in $\mathcal{U}$

$$
U=\bigotimes_{j \in \mathbf{N}} e^{i a / d^{j}}, \quad V=\bigotimes_{j \in \mathbf{N}} e^{i b / d^{j}}
$$

The unital $C^{*}$-algebra $\tilde{\mathcal{U}} \subset \mathcal{U}$ generated by $U$ and $V$ is shift invariant and the following formula justifies the name quantum expanding map for the dynamical system $(\tilde{\mathcal{U}}, \Lambda)$

$$
\Lambda(U)=U^{d}, \Lambda(V)=V^{d}
$$

Therefore we can consider a $C^{*}$-algebraic dynamical system $(\tilde{\mathcal{U}}, \Lambda)$ with a shift invariant trace $\tau$ and a subalgebra of smooth elements $\tilde{\mathcal{U}}_{\infty}$ which consists of linear combinations of monomials in $U, U^{-1}, V, V^{-1}$ with rapidly decreasing coefficients. As $\tilde{\mathcal{U}}$ is generated by two unitaries and hence can be interpreted to a two dimensional "quantum manifold" (a kind of "quantum torus" but different from the irrational rotation algebra) we seek for a natural bilinear form $\Delta$ on $\mathbf{C}^{2} \otimes \tilde{\mathcal{U}}_{\infty}$. It can be defined as

$$
\Delta\left(e_{i} \otimes X, e_{j} \otimes Y\right)=\lim _{n \rightarrow \infty} d^{2 n} \tau\left(D_{i}^{(n)}\left(X^{*}\right), D_{j}^{(n)}(Y)\right) \quad i, j=1,2
$$

where

$$
D_{1}^{(n)}(X)=i\left[a^{(n)}, X\right], D_{2}^{(n)}(X)=i\left[b^{(n)}, X\right]
$$

and $a^{(n)}=1 \otimes \cdots \otimes a \otimes 1 \otimes \cdots$ with the matrix $a$ placed at the site $n$, etc. Putting as $X$ and $Y$ the monomials in $U, U^{-1}, V, V^{-1}$ one can easily check that the limit in Eq.(12) exists and can be extended to the whole smooth algebra $\tilde{\mathcal{U}}_{\infty}$. Similar explicit computation taking into account the Eqs.(11-13) shows that independently of $\xi$ the Lyapunov exponent is equal to $\ln d$. These results are summarized by the following theorem.

THEOREM 1. For any $X, Y \in \tilde{\mathcal{U}}_{\infty}$ the limit in $E q(12)$ exists and defines a nonnegative bilinear form on $\mathbf{C}^{2} \otimes \tilde{\mathcal{U}}_{\infty}$. For any $X \in \tilde{\mathcal{U}}_{\infty}$ and any $\xi \in \mathbf{C}^{2}$ such $\Delta(\xi \otimes X, \xi \otimes X)>0$ the limit in Eq.(5) exists and is equal to $\ln d$.

It follows from Theorem 1 that we obtain a doubly degenerated Lyapunov exponent equal to $\ln d$ and hence the sum of Lyapunov exponents is equal to the Alf entropy.

In the general case of a $C^{*}$-algebraic dynamical system $(\mathcal{A}, \Theta, \omega)$ one can consider its Hilbert space representation. Let $\mathcal{H}_{\omega}$ be a Hilbert space, $\Psi_{\omega} \in \mathcal{H}_{\omega}$ a cyclic separating vector and $\pi: \mathcal{A} \mapsto B\left(\mathcal{H}_{\omega}\right)$ a faithful representation obtained by means of the GNS representation. The state $\omega$ is represented as $\omega(A)=<\Psi_{\omega}, \pi(A) \Psi_{\omega}>$ and the dynamical map is implemented by the isometry $W_{\Theta}$ acting on $\mathcal{H}_{\omega}, \pi(\Theta(X))=W_{\Theta} \pi(X) W_{\Theta}^{*}$ and satisfying $W_{\Theta} \Psi_{\omega}=\Psi_{\omega}$. The von Neumann algebra generated by a given subalgebra $\mathcal{C} \subset B\left(\mathcal{H}_{\omega}\right)$ will be denoted by $\mathcal{M}(\mathcal{C})$. The triple $\left\{\mathcal{M}(\pi(\mathcal{A})), W_{\Theta}, \Psi_{\omega}\right\}$ is called a $W^{*}-$ dynamical system.

Theorem 2 shows the isomorphism, on the level of $W^{*}$-dynamical systems, between the quantum Bernoulli shift and the constructed quantum expanding map.

TheOrem 2. The triple $\left(\mathcal{M}(\pi(\tilde{\mathcal{U}})), W_{\Lambda}, \Psi_{\tau}\right)$ is a $W^{*}$-dynamical system isomorphic to a $W^{*}$-dynamical system $\left(\mathcal{M}(\pi(\mathcal{U})), W_{\Lambda}, \Psi_{\tau}\right)$.

Proof. As $\Lambda$ leaves $\tilde{\mathcal{U}}$ invariant then $W_{\Lambda} \mathcal{M}(\pi(\tilde{\mathcal{U}})) W_{\Lambda}^{*} \subset \mathcal{M}(\pi(\tilde{\mathcal{U}}))$ and $(\mathcal{M}(\pi(\tilde{\mathcal{U}}))$, $\left.W_{\Lambda}, \Psi_{\tau}\right)$ is a $W^{*}$-dynamical system. Obviously $\mathcal{M}(\pi(\tilde{\mathcal{U}})) \subset \mathcal{M}(\pi(\mathcal{U}))$. The von Neumann 
algebra $\mathcal{M}(\pi(\mathcal{U}))$ is generated by the elements $\left\{\pi\left(\Lambda^{j}(u)\right), \pi\left(\Lambda^{j}(v)\right) ; j \in \mathbf{N}\right\}$ where we use the convention that $u$ and $v$ live on the site $j=0$. Hence it is enough to show that $\pi(u), \pi(v) \in \mathcal{M}(\pi(\tilde{\mathcal{U}}))$. But $\pi(u)$ and analogously $\pi(v)$ can be expressed in terms of operations which are allowed within the von Neumann algebra. Namely

$$
\pi(u)=\pi(U)\left[\pi\left(\Lambda\left(U^{*}\right)\right)\right]^{1 / d}
$$

The example discussed in this note provides another supporting argument for the usefulness of the Alf dynamical entropy. It seems that our approach is the only from the existing ones which allows the relations between the quantum Lyapunov exponents and the noncommutative dynamical entropy suggested by the classical theory. The necessity of investigation of (often hidden) noncommutative geometry [5] of quantum dynamical systems is another obvious conclusion.

Acknowledgments. This contribution is based on the pleasant and successful collaboration with M. Fannes, J. Andries and P. Tuyls from K.U. Leuven.

\section{References}

[1] J. Andries and M. DeCock, Dynamical entropy of a non-commutative version of the phase doubling, this volume.

[2] J. Andries, M. Fannes, P. Tuyls, and R. Alicki, The dynamical entropy of the quantum Arnold cat map, Lett. Math. Phys. 35 (1995), 375-383.

[3] R. Alicki and M. Fannes, Defining quantum dynamical entropy, Lett. Math. Phys. 32 (1994), 75-82.

[4] R. Alicki, J. Andries, M. Fannes and P. Tuyls, An algebraic approach to the Kolmogorov-Sinai entropy, Rev. Math. Phys. 8 (1996), 167-184.

[5] A. Connes, Non-commutative Geometry, Academic Press, New York, 1994.

[6] A. Connes, H. Narnhofer, and W. Thirring, Dynamical entropy of $C^{*}$-algebras and von Neumann algebras, Commun. Math. Phys. 112 (1987) 691-719.

[7] I. P. Cornfeld, S. V. Fomin, and Ya. G. Sinai, Ergodic theory, Springer, Berlin, 1982.

[8] G. Emch, H. Narnhofer, W. Thirring, and G.L. Sewell, Anosov actions on noncommutative algebras, J. Math. Phys. 35, (1994) 5582-5599.

[9] T. Hudetz, Quantum dynamical entropy revised, this volume.

[10] G. Lindblad, Dynamical Entropy for Quantum Systems, in: Quantum Probability and Applications, Vol.III, L. Accardi and W. von Waldenfels (eds.), Springer LNM 1303, Berlin, 1988, 183-191.

[11] W. A. Majewski and M. Kuna, On quantum characteristic exponents, J. Math. Phys. 34 (1993) 5007-5015.

[12] P. Tuyls, Towards Quantum Kolmogorov-Sinai Entropy, Ph.D. Thesis, Leuven , 1997.

[13] D. Voiculescu, Dynamical approximation entropies and topological entropy in operator algebras, Commun. Math. Phys. 144, (1992) 443-490. 